\title{
Strong correlation between the rates of intrinsically antibiotic-resistant species and the rates of acquired resistance in Gram-negative species causing bacteraemia, EU/EEA, 2016
}

Vincent Jarlier ${ }^{1,2}$, Liselotte Diaz Högberg ${ }^{3}$, Ole E Heuer 3 , José Campos4, Tim Eckmanns5, Christian G Giske ${ }^{6,7}$, Hajo Grundmann ${ }^{8}$, Alan P Johnson', Gunnar Kahlmeter ${ }^{10}$, Jos Monen ${ }^{11}$, Annalisa Pantosti ${ }^{12}$, Gian Maria Rossolini ${ }^{13,14}$, Nienke van de Sande-

Bruinsma $^{15}$, Alkiviadis Vatopoulos ${ }^{16}$, Dorota Żabicka ${ }^{17}$, Helena Žemličkováa ${ }^{18,19}$, Dominique L Monnet ${ }^{3}$, Gunnar Skov Simonsen ${ }^{20,21}$, EARS-Net participants ${ }^{22}$

1. Sorbonne Universités (Paris 06) Inserm Centre d'Immunologie et des Maladies Infectieuses (CIMI), UMR 1135, Paris, France

2. Assistance Publique - Hôpitaux de Paris, Pitié-Salpêtrière hospital, Laboratoire de Bactériologie-Hygiène, Paris, France

3. European Centre for Disease Prevention and Control, Solna, Sweden

4. Reference and Research Laboratory on Antimicrobial Resistance, Centro Nacional de Microbiología, Instituto de Salud Carlos III, Madrid, Spain

5. Robert Koch Institute, Department for Infectious Disease Epidemiology, Berlin, Germany

6. Department of Laboratory Medicine, Karolinska Institute, Stockholm, Sweden

7. Department of Clinical Microbiology, Karolinska University Hospital, Stockholm, Sweden

8. Medical Center - University of Freiburg, Department for Infection Prevention and Hospital Epidemiology, Freiburg, Germany

9. National Infection Service, Public Health England, London, United Kingdom

10. Clinical Microbiology, Central Hospital, Växjö, Sweden

11. National Institute for Public Health and the Environment, Bilthoven, the Netherlands

12. Department of Infectious Diseases, Istituto Superiore di Sanità, Rome, Italy

13. Department of Experimental and Clinical Medicine, University of Florence, Italy

14. Microbiology and Virology Unit, Florence Careggi University Hospital, Florence, Italy

15. Pan American Health Organization/World Health Organization (PAHO/ WHO), Washington DC, United States

16. Department of Public Health Policy, School of Public Health, University of West Attica, Athens, Greece

17.17 Department of Epidemiology and Clinical Microbiology, National Medicines Institute, Warsaw, Poland

18. National Institute of Public Health, National Reference Laboratory for Antibiotics, Prague, Czech Republic

19. Department of Clinical Microbiology, Faculty of Medicine and University Hospital, Charles University, Hradec Kralove, Czech Republic

20. Department of Microbiology and Infection Control, University Hospital of North Norway, Troms $\emptyset$, Norway

21. Research Group for Host-Microbe Interaction, Faculty of Health Sciences, UiT - The Arctic University of Norway, Troms $\varnothing$, Norway

22. The members of the group are listed at the end of the article

Correspondence: Liselotte Diaz Högberg(liselotte.diaz-hogberg@ecdc.europa.eu)

Citation style for this article:

Jarlier Vincent, Diaz Högberg Liselotte, Heuer Ole E, Campos José, Eckmanns Tim, Giske Christian G, Grundmann Hajo, Johnson Alan P, Kahlmeter Gunnar,
Monen Jos, Pantosti Annalisa, Rossolini Gian Maria, van de Sande-Bruinsma Nienke, Vatopoulos Alkiviadis, Żabicka Dorota, Žemličková Helena, Monnet

Dominique L, Simonsen Gunnar Skov, EARS-Net participants. Strong correlation between the rates of intrinsically antibiotic-resistant species and the rates of

acquired resistance in Gram-negative species causing bacteraemia, EU/EEA, 2016. Euro Surveill. 2019;24(33):pii=1800538. https://doi.org/10.2807/1560-7917.

ES.2019.24.33.1800538

Article submitted on 03 Oct 2018 / accepted on 01 Apr 2019 / published on 15 Aug 2019

Background: Antibiotic resistance, either intrinsic or acquired, is a major obstacle for treating bacterial infections. Aim: Our objective was to compare the countryspecific species distribution of the four Gram-negative species Escherichia coli, Klebsiella pneumoniae, Pseudomonas aeruginosa and Acinetobacter species and the proportions of selected acquired resistance traits within these species. Method: We used data reported for 2016 to the European Antimicrobial Resistance Surveillance Network (EARS-Net) by 30 countries in the European Union and European Economic Area. Results: The country-specific species distribution varied considerably. While $E$. coli accounted for $31.9 \%$ to $81.0 \%$ (median: $69.0 \%$ ) of all reported isolates, the two most common intrinsically resistant species $P$. aeruginosa and Acinetobacterspp. combined (PSEACI) accounted for $5.5 \%$ to $39.2 \%$ of isolates (median: 10.1\%). Similarly, large national differences were noted for the percentages of acquired non-susceptibility to third-generation cephalosporins, carbapenems and fluoroquinolones. There was a strong positive rank correlation between the countryspecific percentages of PSEACI and the percentages of non-susceptibility to the above antibiotics in all four species (rho>0.75 for 10 of the 11 pairs of variables tested). Conclusion: Countries with the highest proportion of $P$. aeruginosa and Acinetobacter spp. were also those where the rates of acquired non-susceptibility in all four studied species were highest. The differences are probably related to national differences in antibiotic consumption and infection prevention and control routines. 


\section{Introduction}

Bloodstream infections constitute a major disease burden in Europe. Gram-negative bacteria such as Escherichia coli, Klebsiella pneumoniae, Pseudomonas aeruginosa and Acinetobacter species are the most common organisms involved in these infections. Data from the European Antimicrobial Resistance Surveillance Network (EARS-Net) show that the proportional contribution of each bacterial species to the total number of blood isolates varies considerably between countries [1]. In particular Acinetobacter spp. and $P$. aeruginosa seem to be proportionally far more commonly isolated in some European countries than in others. Several studies have previously made the same observation [2-4].

Antibiotic resistance is a major obstacle for treating these serious infections. Resistance can be both intrinsic, i.e. due to a species' innate ability to resist a particular antibiotic because of structural or functional characteristics, or acquired though a range of resistance mechanisms emerging either by mutation or acquisition of novel genes.

Among Gram-negative bacteria, intrinsic resistance varies markedly between species. For example, even wild-type isolates of $K$. pneumoniae are intrinsically resistant to penicillins whereas wild-type isolates of $E$. coli are susceptible to the same antibiotics. More importantly, every wild-type isolate of Acinetobacter spp. and $P$. aeruginosa is intrinsically resistant to numerous groups of antibiotics (e.g. aminopenicillin- $\beta$-lactamase inhibitor combinations, first- and second-generation cephalosporins, cefotaxime/ceftriaxone, ertapenem, trimethoprim, tetracyclines and older quinolones) [5], immediately excluding these as possible treatment alternatives. Acquisition of additional resistance traits can further reduce available treatment options, jeopardise the use of major remaining groups of antibiotics including $\beta$-lactams, fluoroquinolones and aminoglycosides. Bacteria can acquire multiple resistance mechanisms, leading to multidrug-resistant (MDR), extensively drug-resistant (XDR) or even pandrugresistant (PDR) isolates [6]. Thus the overall pattern of resistance as presented in the laboratory reports reflects the intrinsic resistance characteristics of the species combined with any additional resistance trait acquired by the isolate.

Similar to the differences in the distribution of Gramnegative species between European countries, EARS-Net has also documented large differences in the percentage of acquired antibiotic resistance $[1,2]$. However, there is no study addressing the possible link between the ranking of the various species of Gram-negative bacteria and the percentage of acquired resistances in these species. As an example, most publications on resistance in $P$. aeruginosa and Acinetobacter spp. only focus on acquired resistance mechanisms or on clonal expansion of resistant epidemic clones, and a few others describe the intrinsic resistance characteristics of these species [7-10]. Correlation between intrinsic and acquired resistance is mentioned only from a mechanistic perspective, such as the link between inducible and de-repressed production of chromosomal AmpC $\beta$-lactamase [8], but not from a statistical or epidemiological point of view.

The objective of the present study was to assess the association between the proportion of the two most common intrinsically resistant species ( $P$. aeruginosa and Acinetobacter spp.) among the four major Gram-negative species ( $E$. coli, K. pneumoniae, $P$. aeruginosa and Acinetobacter spp.) and the percentage of selective acquired resistance traits in these species. As the data source, we used data reported to EARS-Net for countries in the European Union (EU) and European Economic Area (EEA) in the year 2016.

\section{Methods}

\section{Data source and inclusion criteria}

EARS-Net is a surveillance network which collects and analyses data from routine antibiotic susceptibility testing (AST) of bacterial pathogens from all $28 \mathrm{EU}$ countries (Austria, Belgium, Bulgaria, Croatia, Cyprus, the Czech Republic, Denmark, Estonia, Finland, France, Germany, Greece, Hungary, Ireland, Italy, Latvia, Lithuania, Luxembourg, Malta, the Netherlands, Poland, Portugal, Romania, Slovakia, Slovenia, Spain, Sweden and the United Kingdom) and two EEA countries (Iceland and Norway). The network is coordinated by the European Centre for Disease Prevention and Control (ECDC). Only AST results for selected important antibiotics active against invasive bacterial infections are included in EARS-Net. The AST results are ascertained according to agreed protocols [1] and the general quality and comparability of the data are evaluated through an annual external quality assessment exercise distributed to the participating laboratories.

Data on $E$. coli, $K$. pneumoniae, $P$. aeruginosa, and Acinetobacter spp. isolates reported to EARS-Net for the year 2016 were extracted from The European Surveillance System (TESSy) database at ECDC. Data included isolates from blood and cerebrospinal fluid, both considered as markers of bloodstream infections. Data were de-duplicated to only include the first isolate per species, patient and year. For this study, only data from laboratories reporting observations for at least three of the four above-mentioned species were included. The study was limited to only include antibiotics commonly used for first-line treatment of bacteremia caused by Gram-negative species and routinely included in susceptibility testing in most local clinical laboratories in Europe. The AST information for the following antibiotic-species combinations were included in the study dataset: third-generation cephalosporins (ceftriaxone, ceftazidime or cefotaxime for $E$. coli, and $K$. pneumoniae; ceftazidime for $P$. aeruginosa), carbapenems (meropenem or imipenem, for all four species) and 
TABLE 1

Number of reported isolates $(n=176,082)$ and included isolates $(n=173,540)$ of the four targeted species, and percentage of total per country and species, EU/EEA, 2016

\begin{tabular}{|c|c|c|c|c|c|c|c|c|c|c|}
\hline \multirow[t]{2}{*}{ Country } & \multirow{2}{*}{$\begin{array}{l}\text { Isolates } \\
\text { reported to } \\
\text { EARS-Net } \\
\text { N }\end{array}$} & \multirow{2}{*}{$\begin{array}{l}\text { Isolates } \\
\text { included in } \\
\text { this study } \\
\text { n }\end{array}$} & \multicolumn{2}{|c|}{ Escherichia coli } & \multicolumn{2}{|c|}{$\begin{array}{c}\text { Klebsiella } \\
\text { pneumoniae }\end{array}$} & \multicolumn{2}{|c|}{$\begin{array}{c}\text { Pseudomonas } \\
\text { aeruginosa }\end{array}$} & \multicolumn{2}{|c|}{ Acinetobacterspecies } \\
\hline & & & $\mathrm{n}$ & $\%$ & $\mathrm{n}$ & $\%$ & $\mathrm{n}$ & $\%$ & $\mathrm{n}$ & $\%$ \\
\hline Austria & 7,310 & 7,300 & 5,276 & 72.3 & 1,247 & 17.1 & 696 & $9 \cdot 5$ & 81 & 1.1 \\
\hline Belgium & 4,970 & 4,610 & 3,538 & 76.7 & 669 & $14 \cdot 5$ & 334 & 7.2 & 69 & 1.5 \\
\hline Bulgaria & 564 & 479 & 190 & 39.7 & 146 & 30.5 & 56 & 11.7 & 87 & 18.2 \\
\hline Croatia & 1,810 & 1,771 & 1,020 & 57.6 & 312 & 17.6 & 259 & 14.6 & 180 & 10.2 \\
\hline Cyprus & 317 & 317 & 149 & 47.0 & 75 & 23.7 & 64 & 20.2 & 29 & 9.1 \\
\hline Czech Republic & 4,982 & 4,818 & 3,026 & 62.8 & 1,304 & 27.1 & 431 & 8.9 & 57 & 1.2 \\
\hline Denmark & 6,535 & 6,535 & 4,847 & 74.2 & 1,156 & 17.7 & 460 & 7.0 & 72 & 1.1 \\
\hline Estonia & 949 & 905 & 667 & $73 \cdot 7$ & 174 & 19.2 & 56 & 6.2 & 8 & 0.9 \\
\hline Finland & 5,983 & 5,983 & 4,833 & 80.8 & 770 & 12.9 & 352 & 5.9 & 28 & 0.5 \\
\hline France & 16,387 & 16,387 & 11,337 & 69.2 & 2,608 & $15 \cdot 9$ & 1,988 & 12.1 & 454 & 2.8 \\
\hline Germany & 20,359 & 20,186 & 15,619 & 77.4 & 2,809 & 13.9 & 1,320 & 6.5 & 438 & 2.2 \\
\hline Greece & 4,097 & 4,095 & 1,305 & 31.9 & 1,183 & 28.9 & 704 & 17.2 & 903 & 22.1 \\
\hline Hungary & 3,859 & 3,840 & 1,990 & 51.8 & 720 & 18.8 & 731 & 19.0 & 399 & 10.4 \\
\hline Iceland & 237 & 237 & 192 & 81.0 & 25 & 10.5 & 17 & 7.2 & 3 & 1.3 \\
\hline Ireland & 3,755 & 3,597 & 2,855 & 79.4 & 439 & 12.2 & 240 & 6.7 & 63 & 1.8 \\
\hline Italy & 10,339 & 9,703 & 5617 & 57.9 & 2,191 & 22.6 & 1,207 & 12.4 & 688 & 7.1 \\
\hline Latvia & 446 & 393 & 218 & $55 \cdot 5$ & 85 & 21.6 & 16 & 4.1 & 74 & 18.8 \\
\hline Lithuania & 1,284 & 1,265 & 783 & 61.9 & 321 & 25.4 & 74 & 5.8 & 87 & 6.9 \\
\hline Luxembourg & 545 & 545 & 419 & 76.9 & 78 & $14 \cdot 3$ & 40 & $7 \cdot 3$ & 8 & 1.5 \\
\hline Malta & 477 & 477 & 328 & 68.8 & 102 & 21.4 & 40 & 8.4 & 7 & 1.5 \\
\hline Netherlands & 8,184 & 7,841 & 6,123 & 78.1 & 1,067 & 13.6 & 543 & 6.9 & 108 & 1.4 \\
\hline Norway & 4,689 & 4,689 & 3,618 & 77.2 & 811 & $17 \cdot 3$ & 227 & 4.8 & 33 & 0.7 \\
\hline Poland & 4,674 & 4,557 & 2,641 & 58.0 & 1,128 & 24.8 & 403 & 8.8 & 385 & 8.4 \\
\hline Portugal & 9,575 & 9,513 & 5,740 & 60.3 & 2,338 & 24.6 & 1,229 & 12.9 & 206 & 2.2 \\
\hline Romania & 1,017 & 956 & 403 & 42.2 & 328 & $34 \cdot 3$ & 82 & 8.6 & 143 & 15.0 \\
\hline Slovakia & 1,601 & 1,570 & 807 & 51.4 & 458 & 29.2 & 191 & 12.2 & 114 & $7 \cdot 3$ \\
\hline Slovenia & 1,890 & 1,890 & 1,420 & 75.1 & 267 & 14.1 & 143 & 7.6 & 60 & 3.2 \\
\hline Spain & 9,429 & 9,389 & 6,761 & 72.0 & 1,679 & 17.9 & 843 & 9.0 & 106 & 1.1 \\
\hline Sweden & 9,066 & 8,975 & 6,921 & 77.1 & 1,495 & 16.7 & 473 & 5.3 & 86 & 1.0 \\
\hline United Kingdom & 30,752 & 30,717 & 23,685 & 77.1 & 4,232 & 13.8 & 2,186 & 7.1 & 614 & 2.0 \\
\hline Total & 176,082 & 173,540 & 122,328 & 70.5 & 30,217 & 17.4 & 15,405 & 8.9 & 5,590 & 3.2 \\
\hline
\end{tabular}

EARS-Net: European Antimicrobial Resistance Surveillance Network; EU/EEA: European Union and European Economic Area.

${ }^{a}$ Limited to laboratories reporting observations for at least three of the four species $E$. coli, K. pneumoniae, $P$. aeruginosa and Acinetobacter spp.

fluoroquinolones (ciprofloxacin, levofloxacin or ofloxacin for E. coli and $K$. pneumoniae; ciprofloxacin or levofloxacin for $P$. aeruginosa and Acinetobacter spp.) Isolates were considered as non-susceptible when reported as either intermediately susceptible (I) or resistant $(R)$.

Of the 829 laboratories that reported data on any of the targeted species to EARS-Net for the year 2016 (total: 176,082 isolates), 749 fulfilled the inclusion criterion (total: 173,540 isolates) and were included in the final analysis.

\section{Statistical analysis}

The percentages of E. coli, $K$. pneumoniae, $P$. aeruginosa, and Acinetobacter spp. isolates among the total of isolates included in the study, as well as the sum of the percentages of $P$. aeruginosa and Acinetobacter spp. (PSEACI) were calculated for each country. Rank comparison of the percentages of acquired non-susceptibility in each of the four Gram-negative species with the percentage of PSEACI was performed using the Spearman's rank correlation coefficient to assess the monotonic relationships and limit impact of outliers. 
TABLE 2

Percentage of non-susceptible isolates (I or R) per country, antibiotic group and species, EU/EEA, 2016 ( $\mathrm{n}=173,540)$

\begin{tabular}{|c|c|c|c|c|c|c|c|c|c|c|c|c|c|}
\hline \multirow[b]{2}{*}{ Country } & \multicolumn{3}{|c|}{ Escherichia coli } & \multicolumn{3}{|c|}{$\begin{array}{l}\text { Klebsiella } \\
\text { pneumoniae }\end{array}$} & \multicolumn{3}{|c|}{$\begin{array}{c}\text { Pseudomonas } \\
\text { aeruginosa }\end{array}$} & \multicolumn{2}{|c|}{ Acinetobacterspecies } & \multirow{2}{*}{$\begin{array}{c}\text { Composite } \\
\% \text { IR to } \\
\text { broad- } \\
\text { spectrum } \\
\beta \text {-lactams }\end{array}$} & \multirow[b]{2}{*}{$\begin{array}{l}\text { Composite } \\
\% \text { IR to } \mathrm{FQ}^{\mathrm{b}}\end{array}$} \\
\hline & $\begin{array}{l}\% \text { IR to } \\
3 G C\end{array}$ & $\begin{array}{l}\% \text { IR } \\
\text { to } \\
\text { car }\end{array}$ & $\begin{array}{c}\% \text { IR } \\
\text { to } \\
\text { FQ }\end{array}$ & $\begin{array}{l}\% \text { IR } \\
\text { to } \\
3 G C\end{array}$ & $\begin{array}{c}\% \text { IR } \\
\text { to } \\
\text { car }\end{array}$ & $\begin{array}{c}\% \text { IR } \\
\text { to } \\
\text { FQ }\end{array}$ & $\begin{array}{l}\% \text { IR } \\
\text { to } \\
3 G C\end{array}$ & $\begin{array}{l}\% \text { IR } \\
\text { to } \\
\text { car }\end{array}$ & $\begin{array}{c}\% \text { IR } \\
\text { to } \\
\text { FQ }\end{array}$ & $\begin{array}{c}\% \text { IR to } \\
\text { car }\end{array}$ & $\begin{array}{c}\% \text { IR to } \\
\text { FQ }\end{array}$ & & \\
\hline Austria & 10.4 & $<0.1$ & 20.5 & 10.6 & 0.9 & 11.8 & 11.6 & 17.0 & 9.1 & 13.6 & 16.0 & 11.1 & 17.9 \\
\hline Belgium & 11.1 & 0.1 & 25.4 & 23.5 & 3.0 & 27.1 & 8.3 & 12.6 & 17.1 & 1.5 & 11.8 & 12.9 & 24.8 \\
\hline Bulgaria & 41.0 & 1.1 & 43.3 & 75.9 & 6.9 & 64.8 & 38.9 & 33.9 & 35.7 & 77.0 & 64.4 & 57.4 & 52.8 \\
\hline Croatia & 15.6 & 0.0 & 29.4 & 50.0 & 1.9 & 46.3 & 20.5 & 47.9 & 39.9 & 95.0 & 94.8 & 34.4 & 40.4 \\
\hline Cyprus & 30.2 & 0.7 & 47.0 & 32.0 & 12.0 & 37.3 & 10.9 & 26.6 & 21.9 & 71.4 & 71.4 & 33.5 & 41.8 \\
\hline Czech Republic & 16.2 & 0.1 & 31.6 & 52.7 & 0.4 & 50.7 & 18.6 & 20.5 & 33.0 & 7.0 & 17.5 & 26.4 & 36.7 \\
\hline Denmark & 8.1 & 0.0 & 13.8 & 9.9 & 0.4 & 8.3 & 4.5 & 4.6 & 5.0 & 0.0 & 2.8 & 8.1 & 12.1 \\
\hline Estonia & 10.5 & 0.0 & 14.9 & 34.5 & 0.6 & 35.6 & 17.6 & 22.2 & 5.4 & 37.5 & 40.0 & 16.1 & 18.5 \\
\hline Finland & 7.6 & $<0.1$ & 12.3 & 5.3 & 0.3 & 4.9 & 5.4 & 10.8 & 9.6 & 0.0 & 0.0 & 7.5 & 11.1 \\
\hline France & 12.1 & $<0.1$ & 19.4 & 30.1 & 0.9 & 31.5 & 11.7 & 19.2 & 16.4 & 7.8 & $15 \cdot 7$ & 15.7 & 20.9 \\
\hline Germany & 11.8 & $<0.1$ & 20.6 & 14.3 & 0.7 & 14.9 & 11.0 & 18.0 & 18.8 & 5.4 & 8.5 & 12.4 & 19.4 \\
\hline Greece & 19.0 & 1.5 & 32.5 & 73.2 & 67.1 & 70.4 & 38.6 & 75.1 & 38.5 & 95.6 & 95.9 & 60.8 & 58.1 \\
\hline Hungary & 16.9 & 0.0 & 27.2 & 37.6 & 1.0 & 36.2 & 20.7 & 36.8 & 25.6 & 61.2 & 67.8 & 29.1 & 32.8 \\
\hline Iceland & 4.7 & 0.0 & 10.1 & 0.0 & 0.0 & 0.0 & 0.0 & 5.9 & 23.5 & 0.0 & 0.0 & 4.2 & 10.0 \\
\hline Ireland & 12.4 & $<0.1$ & 24.1 & 15.5 & 0.9 & $15 \cdot 7$ & 12.5 & 12.1 & 15.0 & 0.0 & 3.2 & 12.5 & 22.1 \\
\hline Italy & 31.2 & 0.4 & $45 \cdot 5$ & 59.3 & 37.2 & 60.4 & 23.0 & 28.3 & 30.1 & 79.9 & 80.6 & 40.7 & 49.5 \\
\hline Latvia & $25 \cdot 7$ & 0.0 & 29.6 & 45.9 & 5.9 & 48.2 & 26.7 & 37.5 & 43.8 & $75 \cdot 7$ & 86.7 & 39.9 & 43.5 \\
\hline Lithuania & 15.1 & 0.0 & 20.0 & 57.3 & 0.6 & 55.8 & 10.8 & 21.6 & 16.4 & 85.1 & 87.4 & 31.0 & 33.5 \\
\hline Luxembourg & 13.6 & 0.0 & 29.2 & 35.9 & 1.3 & 42.3 & 10.0 & 19.4 & 22.5 & 0.0 & 25.0 & 17.0 & 30.5 \\
\hline Malta & 14.9 & 0.3 & 42.4 & 22.5 & 8.8 & 37.3 & 12.5 & 12.5 & 15.0 & 42.9 & 42.9 & 16.8 & 39.0 \\
\hline Netherlands & 7.0 & 0.0 & 14.0 & 9.8 & 0.1 & 10.2 & 3.5 & 6.1 & 9.0 & 1.9 & 4.7 & 7.3 & 13.1 \\
\hline Norway & 6.1 & 0.1 & 11.9 & 7.4 & 0.0 & 6.1 & 7.1 & 11.6 & 8.4 & 0.0 & 3.0 & 6.6 & 10.6 \\
\hline Poland & 15.2 & $<0.1$ & 37.2 & 65.4 & 3.9 & 68.1 & 19.5 & 31.5 & 33.3 & 69.6 & 83.1 & 33.7 & 48.5 \\
\hline Portugal & 16.8 & 0.1 & 30.2 & 48.6 & 6.4 & 48.8 & 19.9 & 22.4 & 23.4 & 52.2 & 51.2 & 26.1 & 34.4 \\
\hline Romania & 22.7 & 0.8 & 30.4 & 68.9 & 38.7 & 64.7 & 48.1 & 54.9 & 52.4 & 85.3 & 90.9 & 50.7 & 53.2 \\
\hline Slovakia & 31.2 & 0.0 & 42.0 & 62.4 & 3.3 & 68.1 & 31.1 & 46.2 & 48.4 & 32.4 & 46.5 & 42.2 & 50.8 \\
\hline Slovenia & 13.8 & 0.4 & 25.8 & 25.1 & 0.4 & 34.5 & 17.5 & 23.8 & 22.4 & 45.0 & 55.0 & 17.1 & 27.7 \\
\hline Spain & 15.4 & 0.1 & 33.4 & 23.0 & 3.8 & 24.8 & 15.6 & 24.7 & 27.5 & 64.2 & 68.9 & 18.1 & 31.8 \\
\hline Sweden & 8.8 & 0.2 & 14.6 & 6.0 & 0.4 & 7.0 & 7.4 & 13.6 & 7.0 & 2.4 & 4.7 & 8.5 & 12.8 \\
\hline United Kingdom & 10.0 & 0.1 & 17.1 & 9.9 & 0.6 & 9.5 & 5.3 & 7.3 & 9.8 & 2.6 & 4.8 & 9.6 & 15.3 \\
\hline
\end{tabular}

3GC: third-generation cephalosporins; car: carbapenems; EU/EEA: European Union/European Economic Area; FQ: fluoroquinolones; IR: nonsusceptible isolates.

a Composite percentage of $E$. coli non-susceptible to third-generation cephalosporins, $K$. pneumoniae non-susceptible to third-generation cephalosporins, $P$. aeruginosa non-susceptible to carbapenems and Acinetobacter spp. non-susceptible to carbapenems among all tested $E$. coli, K. pneumoniae, $P$. aeruginosa and Acinetobacter spp. isolates.

${ }^{\mathrm{b}}$ Composite percentage of $E$. coli non-susceptible to fluoroquinolones, $K$. pneumoniae non-susceptible to fluoroquinolones, $P$. aeruginosanonsusceptible to fluoroquinolones and Acinetobacter spp. non-susceptible to fluoroquinolones among all tested $E$. coli, K. pneumoniae, $P$. aeruginosa and Acinetobacter spp. isolates.

All statistical analyses were performed using Stata Statistical Software Release 14 (StataCorp, College Station, United States).

\section{Ethical statement}

Antimicrobial resistance is listed as a special health issue in the EU case definitions for which ECDC routinely collects, analyses and disseminates surveillance data as stated by the Article 3 of its founding regulation. TESSy data are pseudonymised and processed for public interest in the area of public health. Approval of the study by an ethics committee was therefore not necessary.

\section{Results}

Distribution of the species

Overall, E. coli was the most commonly reported species (70.5\%), followed by K. pneumoniae (17.4\%), P. aeruginosa $(8.9 \%)$ and Acinetobacter spp. (3.2\%) 


\section{FIGURE 1}

Distribution of fully susceptible (S) and non-susceptible (I or R) isolates in four Gram-negative species isolated from blood or cerebrospinal fluid, 30 EU/EEA countries, $2016(n=173,540)$

A. Third-generation cephalosporins for Klebsiella pneumoniae and Escherichia coli, and carbapenems for Pseudomonas aeruginosa and Acinetobacter spp.

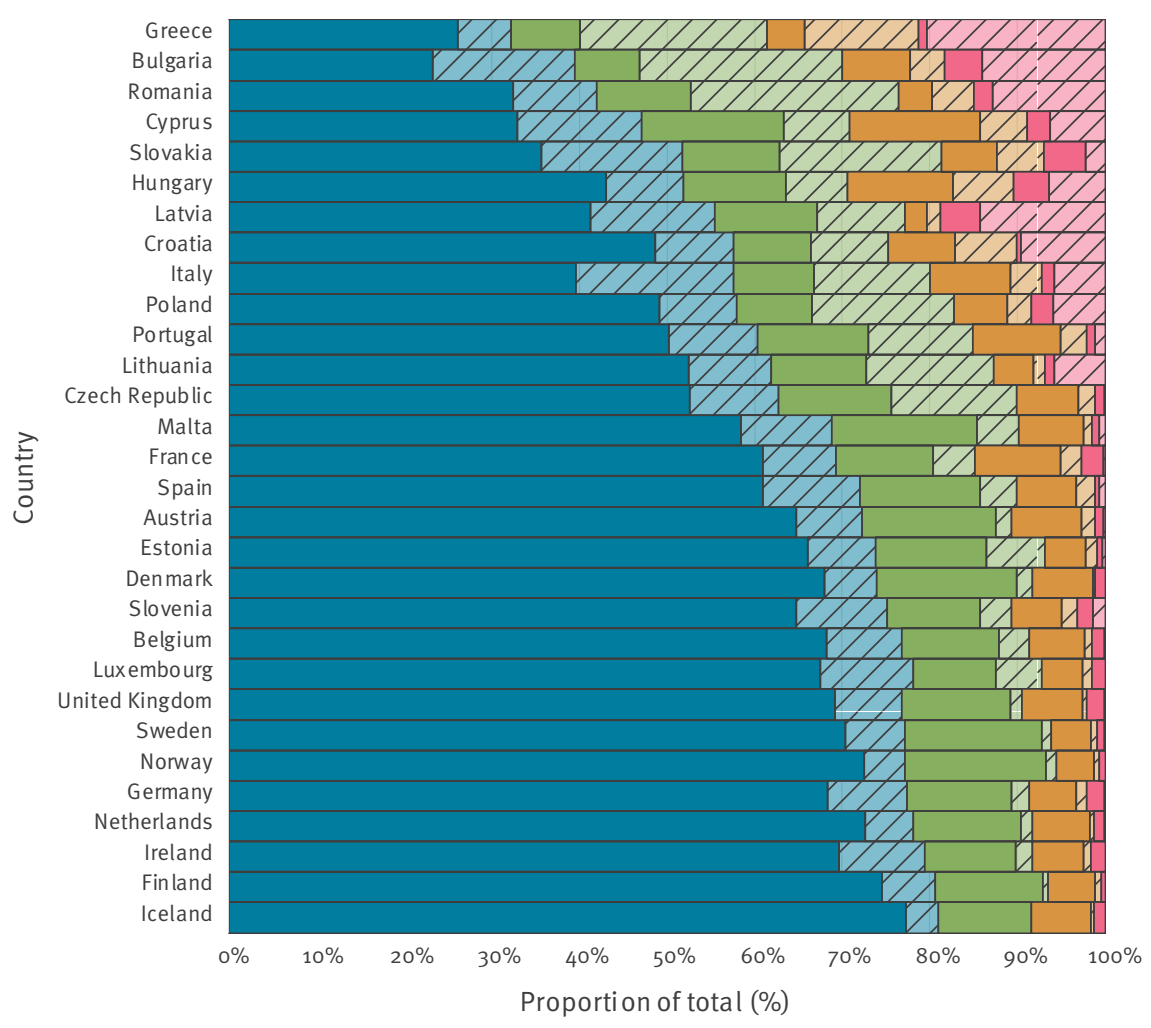

Escherichia coli S to $3 \mathrm{GC}$

$\square$ Escherichia coli IR to $3 \mathrm{GC}$

$\square$ Klebsiella pneumoniae $\mathrm{S}$ to $3 \mathrm{GC}$

$\measuredangle$ Klebsiella pneumoniae IR to $3 \mathrm{GC}$

$\square$ Pseudomonas aeruginosa S to car

ए7seudomonas aeruginosa IR to car

$\square$ Acinetobacter spp. S to car

I Acinetobacter spp. IR to car

\section{B. Fluoroquinolones for the four species}

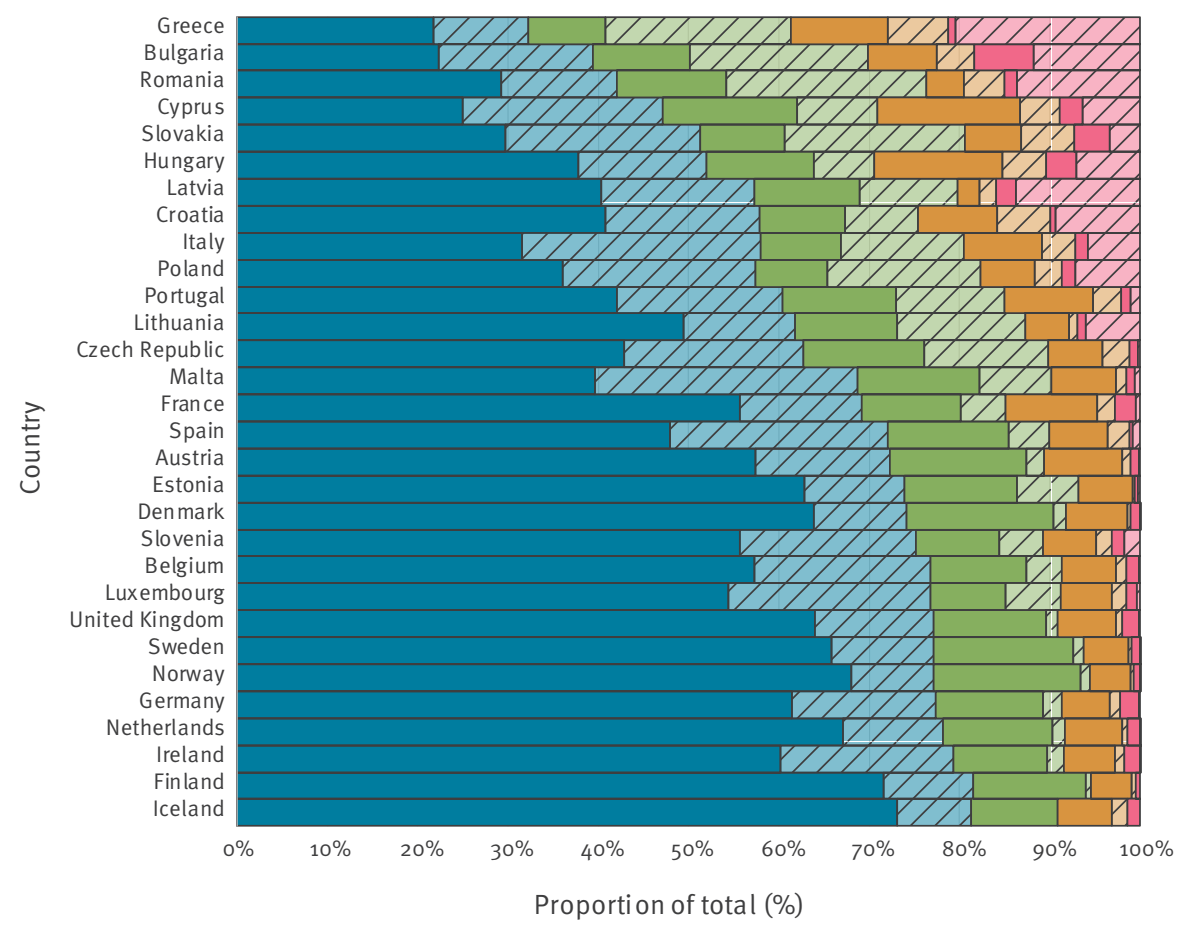

Escherichia coli S to FQ

$\square$ Escherichia coli IR to FQ

$\square$ Klebsiella pneumoniae S to FQ

$\square$ Klebsiella pneumoniae IR to $\mathrm{FQ}$

$\square$ Pseudomonas aeruginosa S to FQ

$\square$ Pseudomonas aeruginosa IR to FQ

$\square$ Acinetobacter spp. S to FQ

$\square$ Acinetobacter spp. IR to FQ

3GC: third-generation cephalosporins; car: carbapenems; EU/EEA: European Union/European Economic Area; FQ: fluoroquinolones; I: intermediately susceptible; R: resistant; S: susceptible.

The countries are ranked by increasing proportion of Escherichia coli isolates. Colour shading: susceptible isolates; hatched areas: nonsusceptible isolates. 
(Table 1). The species distribution varied considerably between countries. Although $E$. coli remained the most commonly reported species in all 30 countries, the percentage of $E$. coli ranged from $31.9 \%$ (Greece) to $81.0 \%$ (Iceland), with a median of $69.0 \%$. For K. pneumoniae, the percentage ranged from $10.5 \%$ (Iceland) to $34.3 \%$ (Romania), with a median of $17.5 \%$. For P. aeruginosa, the percentage ranged from $4.1 \%$ (Latvia) to $20.2 \%$ (Cyprus) (median 7.5\%) and for Acinetobacter spp. from $0.5 \%$ (Finland) to $22.1 \%$ (Greece) (median 1.9\%). The combined percentage of the two PSEACI species among the total number of isolates ranged from $5.5 \%$ (Norway) to $39.2 \%$ (Greece), with a median of $10.1 \%$.

\section{Antibiotic non-susceptibility}

Third-generation cephalosporins

The percentage of isolates with acquired non-susceptibility to third-generation cephalosporins ranged from $4.7 \%$ (Iceland) to $41.0 \%$ (Bulgaria) in E. coli (median: $14.4 \%$ ), from $0.0 \%$ (Iceland) to $75.9 \%$ (Bulgaria) in $K$. pneumoniae (median: $31.1 \%$ ) and from $0.0 \%$ (Iceland) to $48.1 \%$ (Romania) in $P$. aeruginosa (median: $12.5 \%$ ) (Table 2).

\section{Carbapenems}

The percentage of isolates with acquired non-susceptibility to carbapenems ranged from 0.0\% (Croatia, Denmark, Estonia, Hungary, Iceland, Latvia, Lithuania, Luxembourg, the Netherlands and Slovakia) to $1.5 \%$ (Greece) in E. coli(median: $<0.1 \%$ ), from $0.0 \%$ (Iceland and Norway) to $67.1 \%$ (Greece) in K. pneumoniae (median: $1.0 \%$ ), from $4.6 \%$ (Denmark) to $75.1 \%$ (Greece) in P. aeruginosa (median: $21.0 \%$ ) and from $0.0 \%$ (Denmark, Finland, Iceland, Ireland, Luxembourg and Norway) to $95.6 \%$ (Greece) in Acinetobacter spp. (median: $35.0 \%$ ) (Table 2 ).

\section{Fluoroquinolones}

The percentage of isolates with acquired non-susceptibility to fluoroquinolones ranged from $10.1 \%$ (Iceland) to $47.0 \%$ (Cyprus) in E. coli (median: $26.5 \%$ ), from $0 \%$ (Iceland) to $70.4 \%$ (Greece) for K. pneumoniae (median: $35.9 \%$ ), from $5.0 \%$ (Denmark) to $52.4 \%$ (Romania) in $P$. aeruginosa (median: 22.1\%), and from o\% (Finland and Iceland) to $95.9 \%$ (Greece) in Acinetobacter spp. (median: 41.4\%) (Table 2).

Combining the percentages of susceptible (S) and non-susceptible $(I+R)$ isolates in each of the four Gram-negative species

Figure 1 shows, within each of the four Gram-negative species reported by the 30 countries, the distribution of isolates fully susceptible (S) and non-susceptible $(I+R)$ to broad-spectrum $\beta$-lactams (third-generation cephalosporins for $K$. pneumoniae and $E$. coli, carbapenems for $P$. aeruginosa and Acinetobacter spp.) (Figure 1A) and to fluoroquinolones for the four species (Figure 1B). The sum of these non-susceptible isolates, expressed as the composite percentage of isolates intermediately susceptible and resistant to broad-spectrum $\beta$-lactams, ranged from $4.2 \%$ for Iceland and $6.6 \%$ for Norway to $15.7 \%$ for France and $18.1 \%$ for Spain, up to $57.4 \%$ for Bulgaria and $60.8 \%$ for Greece (Table 2). In Figure $1 B$, the composite percentage of isolates intermediately susceptible and resistant to fluoroquinolones ranged from $10.0 \%$ for Iceland and $10.6 \%$ for Norway to $20.9 \%$ for France and $31.8 \%$ for Spain, up to $52.8 \%$ for Bulgaria and $58.1 \%$ for Greece. Among the total of isolates from bloodstream infection involving the four Gram-negative species, the proportion of $E$. coli isolates susceptible to third-generation cephalosporins ranged from $77.5 \%$ in Iceland to only $24.3 \%$ in Bulgaria, whereas the proportion of Acinetobacter spp. isolates non-susceptible to carbapenems ranged from ०\% in Denmark, Finland, Iceland, Ireland, Luxembourg and Norway to $21.7 \%$ in Greece.

\section{Correlation between country-specific} percentages of acquired non-susceptibility in the four species and proportion of Pseudomonas aeruginosa and Acinetobacter spp.

There was a strong positive correlation between the ranks of the country-specific percentages of non-susceptibility to third-generation cephalosporins and the percentage of PSEACl: E. coli $(r h o=0.88, p<0.0001)$ (Figure 2A), K. pneumoniae (rho $=0.82, p<0.0001$ ) and $P$. aeruginosa $(\mathrm{rho}=0.78, \mathrm{p}<0.0001)$. Similar positive correlations were found for non-susceptibility to carbapenems in $K$. pneumoniae $(\mathrm{rho}=0.76$, $\mathrm{p}<0.0001), \quad P$. aeruginosa $(\mathrm{rho}=0.85, \quad \mathrm{p}<0.0001)$ and Acinetobacter spp. (rho $0.85, \mathrm{p}<0.0001$ ). The correlation was more moderate and not statistically significant for non-susceptibility to carbapenems in $E$. coli $(r=0.33, p=0.077)$ (Figure $2 \mathrm{~B})$. Finally, this correlation was also strong for non-susceptibility to fluoroquinolones in $E$. coli $(r h o=0.75, p<0.0001) K$. pneumoniae $(r h o=079, p<0.0001), P$. aeruginosa (rho $=0.79, \quad \mathrm{p}<0.0001)$ and Acinetobacter spp. (rho $=0.85, p<0.0001)$ (Figure $2 \mathrm{C})$.

\section{Discussion}

The intrinsic resistance profiles of different bacteria are reflected in the European Committee on Antimicrobial Susceptibility Testing (EUCAST) expert rules [5] as well as in the number of antimicrobial drugs that must be considered to define acquired MDR/XDR/ PDR patterns [6]. As a consequence, for Gram-negative bacteria, identification of the species isolated from a positive blood culture provides immediate information to the microbiologist and the clinician about which antimicrobials should not be used because of intrinsic resistance in that species. This is one of the major reasons for the development of rapid methods for bacterial species identification, such as matrix-assisted laser desorption/ionization time-of-flight (MALDI-TOF) mass-spectrometry. In addition, acquired resistance is recognised as a major global public health issue $[11,12]$ because it jeopardises the effectiveness of antimicrobial drugs that are normally active against intrinsically multi-susceptible species such as $E$. coli and because it further reduces the possibility to treat 


\section{FIGURE 2}

Scattergrams showing the sum of proportions of Pseudomonas aeruginosa and Acinetobacter spp. combined (PSEACI) ( $\mathrm{n}=$ 20,995) among four Gram-negative species ${ }^{\mathrm{a}}$ and proportions of various acquired non-susceptibility and species, 30 EU/EEA countries, 2016

A. Non-susceptibility to third-generation cephalosporins in Escherichia coli

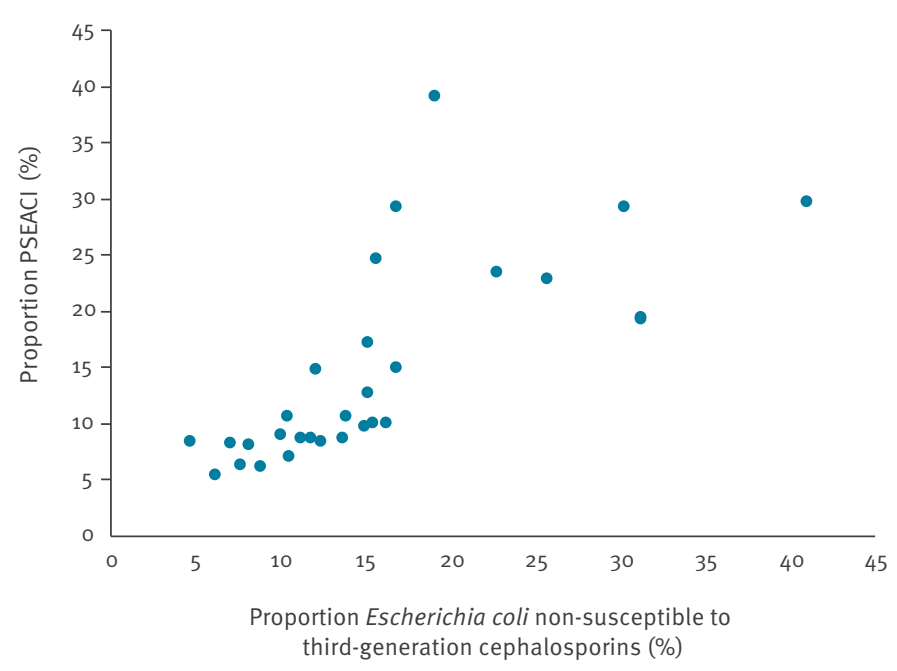

C. Non-susceptibility to fluoroquinolones in Klebsiella pneumoniae

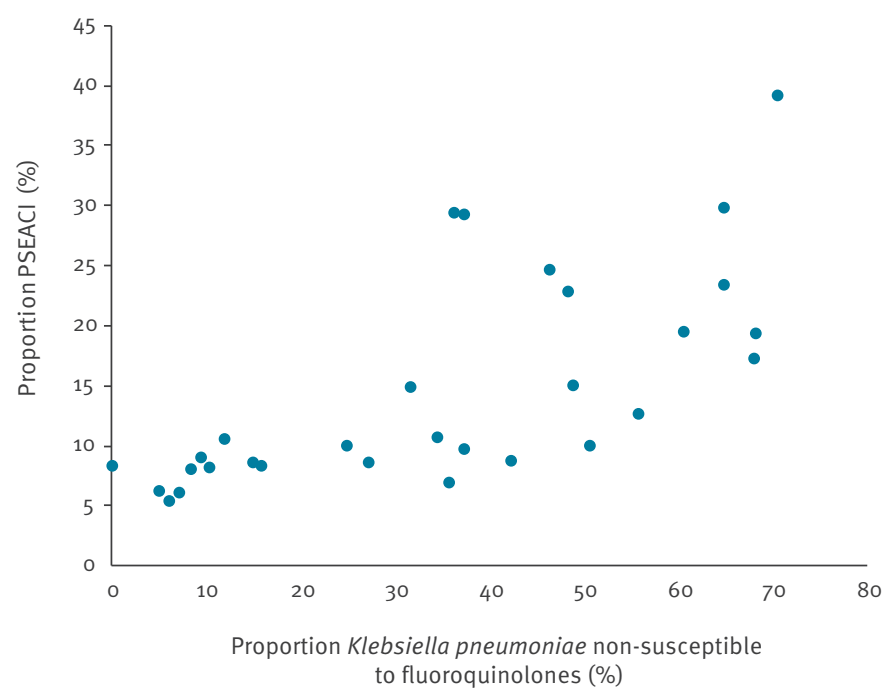

B. Non-susceptibility to carbapenems in Acinetobacter spp.

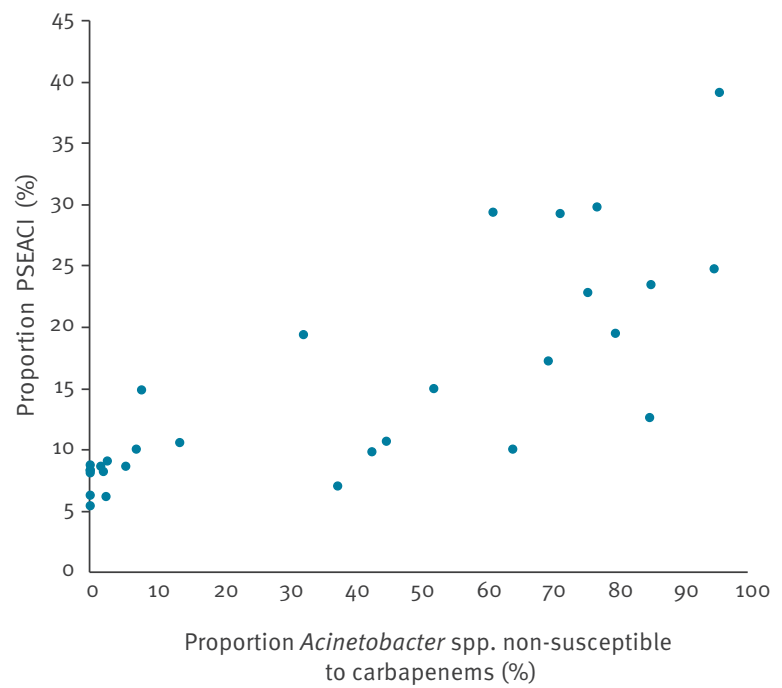

Each dot represents a country.

a Escherichia coli, Klebsiella pneumoniae, Pseudomonas aeruginosa and Acinetobacter spp.

infections with species that are already intrinsically resistant to many antimicrobial drugs such as $P$. aeruginosa and Acinetobacter spp.

The results presented here, based on an analysis of EARS-Net data for the year 2016 and focusing on four major Gram-negative species ( $E$. coli, K. pneumoniae, $P$. aeruginosa and Acinetobacter spp.) isolated from bloodstream infections, clearly show significant statistical association between the distribution of species, and the percentages of acquired nonsusceptibility to major antibiotic groups. All but one correlation were statistically significant, with Spearman's rank correlation coefficient being $>0.75$ for 10 of the 11 pairs of variables tested, which is generally considered as indicating a strong correlation $[13,14]$. In short, the higher the proportion of the two most intrinsically resistant species $P$. aeruginosa and Acinetobacter spp., the higher the percentages of acquired non-susceptibility in $E$. coli, $K$. pneumoniae, $P$. aeruginosa and Acinetobacter spp., a point that directly indicates the burden of intrinsic resistance. Consequently, there were, at one extremity of the EU/EEA gradient, countries with a very low 
proportion of bloodstream infections caused by the two most intrinsically resistant species together with low percentages of isolates with acquired non-susceptibility in any of the four Gram-negative species. At the opposite extremity of the EU/EEA gradient, countries with high percentages of bloodstream isolates caused by the two most intrinsically resistant species had high percentages of isolates with acquired non-susceptibility in all the four species. The weak statistical link noted for carbapenem non-susceptibility in E. coli could be due to very low percentages of non-susceptibility reported from a majority of the countries [1].

Statistical association is not equal to causation and the correlations presented in this study do not mean that there is a cause-and-effect relationship between intrinsic and acquired resistance. However, we can hypothesise that the two major driving forces of antibiotic resistance, i.e. the use of antibiotics acting as a selective pressure on resistant bacteria and the spread of the selected antibiotic-resistant bacteria by cross-transmission between humans, animals and the environment, apply to both the intrinsically resistant species such as $P$. aeruginosa and Acinetobacter spp. (and to a lesser extent $K$. pneumoniae), and to strains with acquired resistance (in the present study: resistance to $\beta$-lactams or fluoroquinolones). Antibiotic use has a strong selective effect on $P$. aeruginosa and Acinetobacter spp $[15,16]$ and on strains with acquired resistance traits in many species [1720]. Host-to-host cross-transmission of $P$. aeruginosa and Acinetobacter spp [21,22] and of strains with acquired resistance traits e.g. in enterobacteria [23,24] also plays a crucial role in the spread of resistance. Indeed, the available comparative data suggest that the EU/EEA countries that in our study had the highest proportion of intrinsically resistant species and percentages of isolates with acquired resistance, generally also have the highest antibiotic consumption in humans (in particular broad-spectrum $\beta$-lactams and fluoroquinolones) and the lowest levels of preventive measures against cross-transmission of microorganisms in hospitals such as consumption of alcohol hand rub solutions, proportion of rooms with a single bed and staffing of infection control teams $[2,25,26]$. Differences in healthcare systems or, possibly, climate issues could also be involved in the discrepancies between countries.

This study has several limitations. Firstly, although a very large number of isolates from bloodstream infections $(n=173,540)$ were analysed, this study only included the four Gram-negative species covered by EARS-Net (E. coli, K. pneumoniae, P. aeruginosa and Acinetobacter spp.) and did not cover other species such as Enterobacter cloacae, Serratia marcescens or Proteus mirabilis. However, the included four species taken together constitute the majority of invasive aerobic Gram-negative isolates in many studies: $78 \%$ of bloodstream infections recorded by the SENTRY surveillance programme organised in Europe from 1997 to 1998 [27], $73 \%$ in the European point prevalence survey coordinated by ECDC in 2011 and 2012 [2] and 74\% in a meta-analysis on infections recorded in developing countries [28]. Secondly, our study did not cover all antibiotics but only a selection of broad-spectrum $\beta$-lactams (third-generation cephalosporins and carbapenems) and the fluoroquinolones, widely used for treating bacteraemia caused Gram-negative species. However, we observed in the same data source similar types of correlations with aminoglycosides, another major class of antibiotics used for treating such severe infections (data not shown). Finally, the patient casemix, which depends on the types of included hospitals and on the frequency of blood culture sampling in each country, might have had an impact on the reported resistance percentages. Importantly, in the EARS-Net reports that provide detailed information on the number of laboratories and characteristics of the hospitals included, there were no marked differences between countries with low and high resistance percentages concerning the proportions of tertiary care hospital beds and intensive care unit beds, two types of hospital settings where resistance rates are usually the highest $[1,29]$. In addition, the representativeness of the population sample for 2016 data has been assessed as high in 23 of the 30 countries [29]. However, there was a trend towards a lower number of blood culture sets taken per 1,000 patient days in some of the countries with the highest percentages of resistance [29], which may have led us to overestimate the percentage of acquired resistance or the percentage of $\mathrm{PSEACl}$ in these countries. Concerning the quality of antibiotic susceptibility testing in EARS-Net, the widespread implementation of EUCAST clinical breakpoints in Europe and the high proportion of laboratories that participated in 2016 in the annual EARS-Net external quality assessment exercises with satisfactory results [1] greatly helps to ascertain the ability of the EU/EEA countries to report robust and trustworthy antimicrobial resistance data to EARS-Net.

\section{Conclusion}

We observed a strong correlation in bloodstream infections between on the one hand the countries with most intrinsically resistant Gram-negative species, indicating the burden of intrinsic resistance, and on the other hand the percentage of acquired non-susceptibility in these species. This important information adds to the already well-established arguments for a strong reduction in the consumption of antibiotics, particularly those with broad-spectrum activity, which exert a selective pressure on all types of resistant bacteria. It also reinforces the crucial importance of measures to prevent host-to-host cross-transmission of antibioticresistant microorganisms, not only to control acquired resistance in every bacterial species but also to limit the burden of infections caused by species such as $P$. aeruginosa and Acinetobacter spp., in which intrinsic resistance per se represents a therapeutic problem. 


\section{EARS-Net participants group}

Austria: Reinhild STRAUSS, Federal Ministry for Labour, Social Affairs, Health and Consumer Protection; Vienna, Austria; Belgium: Karl MERTENS, Sciensano, Brussels, Belgium; Bulgaria: Yuliya Stoyanova MARTEVA-PROEVSKA, Central Laboratory of Clinical Microbiology, University Multiprofile Hospital for Active Treatment (UMHAT), Bulgaria; Croatia: Silvija ŠOPREK, University Clinic for Infectious Diseases “Dr. Fran Mihaljevićc, Zagreb, Department for Clinical Microbiology, Zagreb, Croatia; Cyprus: Panagiota MAIKANTI-CHARALAMPOUS, Microbiology DepartmentNational Reference Laboratory for Antimicrobial Resistance Surveillance, Nicosia General Hospital 215, Nicosia, CYPRUS; Czech Republic: Vladislav JAKUBU, National Institute of Public Health, Prague, Czech Republic; Denmark: Ute SÖNKSEN, Statens Serum Institut, Copenhagen, Denmark; Estonia: Marina IVANOVA, East Tallinn Central Hospital Central Laboratory, Tallinn, Estonia; France: Sylvie MAUGAT, Santé Publique France, the French Public Health Agency, Saint-Maurice, France; Finland: Jari JALAVA, Infectious Disease Control and Vaccinations Unit, National Institute for Health and Welfare, Helsinki, Finland; Germany: Ines NOLL, Healthcare-associated infections, surveillance of antimicrobial resistance and consumption Department for Infectious Disease Epidemiology Robert Koch Institute, Berlin, Germany ; Greece: Michalis POLEMIS, Hellenic National Public Health Organization, Athens, Greece ; Hungary: Zsolt VEGH, Directorate of Clinical and Public Health Microbiology, National Public Health Institute, Budapest, Hungary ; Iceland: Karl G. KRISTINSSON, Clinical Microbiology, Landspitali University Hospital, Reykjavik, Iceland ; Ireland: Stephen MURCHAN, Health Protection Surveillance Centre, Dublin 1, Ireland; Latvia: Arta Olga BALODE, Department of Biology and Microbiology, Rīga Stradinš University, Riga, Latvia; Lithuania: Jolanta MICIULEVICIÉNE , National Public Health Surveillance Laboratory, Vilnius, Lithuania ; Luxembourg: Monique PERRIN, Laboratoire National de Santé, Dudelange, Luxembourg; the Netherlands: Sjoukje H. S. WOUDT, Centre for Infectious Disease Control (Clb), National Institute for Public Health and the Environment (RIVM), Bilthoven, the Netherlands; Norway: Frode W GRAN, St. Olav University Hospital, Trondheim, Norway; Poland: Waleria HRYNIEWICZ, National Medicines Institute, Warsaw, Poland; Portugal: Manuela CANICCA, National Institute of Health Doutor Ricardo Jorge, Lisboa, Portugal; Romania: Andreea Sorina NICULCEA, National Institute of Public Health, Bucharest, Romania; Slovakia: Eva SCHRETEROVA, Louis Pasteur University Hospital Kosice, Kosice, Slovakia; Slovenia: MajaŠUBELJ , National Institute of Public Health, Ljubljana, Slovenia; Spain: Belén ARACIL, Reference and Research Laboratory on Antimicrobial Resistance, Centro Nacional de Microbiología, Instituto de Salud Carlos III, Madrid, Spain; Sweden: Hanna BILLSTRÖM, Public Health Agency of Sweden, Solna, Sweden and The Swedish Antimicrobial Resistance Surveillance Network (Svebar); United Kingdom: Eleanor ANDERSON, Health Protection Scotland - NHS National Services Scotland.

\section{Acknowledgements}

The authors acknowledge the work performed by the staff of the participating clinical microbiology laboratories and of the national healthcare services that provided data to EARS-Net.

\section{Conflict of interest}

None declared.
Authors' contributions

Conceptualisation of the study: VJ. Design of the study: VJ, LDH, GSS. Acquisition and analysis of the data: all authors. Interpretation of results of the study: VJ, LDH, OEH, JC, TE, CGG, HG, APJ, GK, JM, AP, GMR, NSB, AV, DZ, HZ, DLM, GSS. National interpretations were provided by the EARS-Net participants group. Writing of the first draft: VJ, LDH, GSS. All authors critically reviewed and edited the final manuscript.

\section{References}

1. European Centre for Disease Prevention and Control (ECDC). Surveillance of antimicrobial resistance in Europe 2016. Annual Report of the European Antimicrobial Resistance Surveillance Network (EARS-Net). Stockholm: ECDC; 2017. Available from: https://ecdc.europa.eu/sites/portal/files/documents/AMRsurveillance-Europe-2016.pdf

2. European Centre for Disease Prevention and Control (ECDC). Point prevalence survey of healthcare-associated infections and antimicrobial use in European acute care hospitals 2011-2012. Stockholm: ECDC; 2013. Available from: https:// ecdc.europa.eu/sites/portal/files/media/en/publications/ Publications/healthcare-associated-infections-antimicrobialuse-PPS.pdf

3. Falagas ME, Karveli EA, Siempos II, Vardakas KZ. Acinetobacter infections: a growing threat for critically ill patients. Epidemiol Infect. 2008;136(8):1009-19. https://doi.org/10.1017/ So950268807009478 PMID: 17892629

4. Jarlier V, Fosse T, Philippon A. Antibiotic susceptibility in aerobic gram-negative bacilli isolated in intensive care units in 39 French teaching hospitals (ICU study). Intensive Care Med. 1996;22(10):1057-65. https://doi.org/10.1111/j.14690691.2011.03703.x PMID: 22117544

5. Leclercq R, Cantón R, Brown DF, Giske CG, Heisig P, MacGowan $A P$, et al. EUCAST expert rules in antimicrobial susceptibility testing. Clin Microbiol Infect. 2013;19(2):141-60. https://doi. org/10.1111/j.1469-0691.2011.03703.x PMID: 22117544

6. Magiorakos AP, Srinivasan A, Carey RB, Carmeli Y, Falagas ME, Giske CG, et al. Multidrug-resistant, extensively drugresistant and pandrug-resistant bacteria: an international expert proposal for interim standard definitions for acquired resistance. Clin Microbiol Infect. 2012;18(3):268-81. https:// doi.org/10.1111/j.1469-0691.2011.03570.x PMID: 21793988

7. Bonomo RA, Szabo D. Mechanisms of multidrug resistance in Acinetobacter species and Pseudomonas aeruginosa. Clin Infect Dis. 2006;43(Suppl 2):S49-56. https://doi. org/10.1086/504477 PMID: 16894515

8. Hancock REW, Speert DP. Antibiotic resistance in Pseudomonas aeruginosa: mechanisms and impact on treatment. Drug Resist Updat. 2000;3(4):247-55. https://doi.org/10.1054/ drup.2000.0152 PMID: 11498392

9. El Zowalaty ME, Al Thani AA, Webster TJ, El Zowalaty AE, Schweizer HP, Nasrallah GK, et al. Pseudomonas aeruginosa: arsenal of resistance mechanisms, decades of changing resistance profiles, and future antimicrobial therapies. Future Microbiol. 2015;10(10):1683-706. https://doi.org/10.2217/ fmb.15.48 PMID: 26439366

10. Poirel L, Nordmann P. Carbapenem resistance in Acinetobacter baumannii: mechanisms and epidemiology. Clin Microbiol Infect. 2006;12(9):826-36. https://doi.org/10.1111/j.14690691.2006.01456.x PMID: 16882287

11. United Nations (UN). General Assembly of the UN. Political declaration of the high-level meeting of the General Assembly on Antimicrobial Resistance: draft resolution / submitted by the President of the General Assembly. New York: UN; 2016 Available from: https://digitallibrary.un.org/ record $/ 842813$ ? In $=$ en

12. World Health Organization (WHO). Global action plan on antimicrobial resistance. Geneva: WHO; 2015. Available from: http://www.who.int/antimicrobial-resistance/publications/ global-action-plan/en/

13. Taylor R. Interpretation of the correlation coefficient: a basic review. J Diagn Med Sonogr. 1990;6(1):35-9. https://doi. org/10.1177/875647939000600106

14. Spearman's rank-order correlation. Derby: Lærd Statistics. [Accessed: 18 August 2018]. Available from: https://statistics. laerd.com/statistical-guides/spearmans-rank-ordercorrelation-statistical-guide.php

15. Venier AG, Leroyer C, Slekovec C, Talon D, Bertrand X, Parer $S$, et al. Risk factors for Pseudomonas aeruginosa acquisition in intensive care units: a prospective multicentre study. I Hosp Infect. 2014;88(2):103-8. https://doi.org/10.1016/j. jhin.2014.06.018 PMID: 25155240 
16. García-Garmendia JL, Ortiz-Leyba C, Garnacho-Montero J, Jiménez-Jiménez FJ, Pérez-Paredes C, Barrero-Almodóvar AE, et al. Risk factors for Acinetobacter baumannii nosocomial bacteremia in critically ill patients: a cohort study. Clin Infect Dis. 2001;33(7):939-46. https://doi.org/10.1086/322584 PMID: 11528563

17. Lemos EV, de la Hoz FP, Einarson TR, McGhan WF, Quevedo $E$, Castañeda C, et al. Carbapenem resistance and mortality in patients with Acinetobacter baumannii infection: systematic review and meta-analysis. Clin Microbiol Infect. 2014;20(5):416-23. https://doi.org/10.1086/322584 PMID: 11528563

18. Nicolas-Chanoine MH, Petitjean M, Mora A, Mayer N, Lavigne JP, Boulet O, et al. The ST131 Escherichia coli H22 subclone from human intestinal microbiota: Comparison of genomic and phenotypic traits with those of the globally successful H30 subclone. BMC Microbiol. 2017;17(1):71. https://doi. org/10.1186/S12866-017-0984-8 PMID: 28347271

19. Falagas ME, Kopterides P. Risk factors for the isolation of multi-drug-resistant Acinetobacter baumannii and Pseudomonas aeruginosa: a systematic review of the literature. J Hosp Infect. 2006;64(1):7-15. https://doi. org/10.1016/j.jhin.2006.04.015 PMID: 16822583

20. Voor In 't Holt AF, Severin JA, Lesaffre EMEH, Vos MC. A systematic review and meta-analyses show that carbapenem use and medical devices are the leading risk factors for carbapenem-resistant Pseudomonas aeruginosa. Antimicrob Agents Chemother. 2014;58(5):2626-37. https://doi. org/10.1128/AAC.01758-13 PMID: 24550343

21. Agodi A, Barchitta M, Cipresso R, Giaquinta L, Romeo MA, Denaro C. Pseudomonas aeruginosa carriage, colonization, and infection in ICU patients. Intensive Care Med. 2007;33(7):115561. https://doi.org/10.1007/s00134-007-0671-6 PMID: 17503016

22. Doan TN, Kong DC, Marshall C, Kirkpatrick CM, McBryde ES Characterising the transmission dynamics of Acinetobacter baumannii in intensive care units using hidden Markov models. PLoS One. 2015;10(7):e0132037. https://doi.org/10.1371/ journal.pone.0132037 PMID: 26131722

23. Stapleton PJM, Murphy M, McCallion N, Brennan M, Cunney R, Drew RJ. Outbreaks of extended spectrum betalactamase-producing Enterobacteriaceae in neonatal intensive care units: a systematic review. Arch Dis Child Fetal Neonatal Ed. 2016;101(1):F72-8. https://doi.org/10.1136/ archdischild-2015-308707 PMID: 26369370

24. Hendrik TC, Voor In 't Holt AF, Vos MC. Clinical and molecular epidemiology of extended-spectrum beta-lactamase-producing Klebsiella spp.: a systematic review and meta-analyses. PLoS One. 2015;10(10):e0140754. https://doi.org/10.1371/journal. pone.0140754 PMID: 26485570

25. European Centre for Disease Prevention and Control (ECDC). Summary of the latest data on antibiotic consumption in the European Union. ESAC-Net surveillance data, November 2017. Stockholm: ECDC; 2017. Available from: https://ecdc.europa. eu/sites/portal/files/documents/Final_2017_EAAD_ESAC-Net_ Summary-edited\%20-\%20FINALwith\%2oerratum.pdf

26. European Centre for Disease Prevention and Control (ECDC), European Food Safety Authority and European Medicines Agency (EFSA), European Medicines Agency (EMA). ECDC/ EFSA/EMA second joint report on the integrated analysis of the consumption of antimicrobial agents and occurrence of antimicrobial resistance in bacteria from humans and food-producing animals - Joint Interagency Antimicrobial Consumption and Resistance Analysis (JIACRA) Report. EFSA Journal 2017;15(7):4872. https://doi.org/http://dx.doi. org/10.2903/j.efsa.2017.4872 . Available from: https://ecdc. europa.eu/sites/portal/files/documents/efs2_4872_final.pdf

27. Fluit AC, Jones ME, Schmitz FJ, Acar J, Gupta R, Verhoef J. Antimicrobial susceptibility and frequency of occurrence of clinical blood isolates in Europe from the SENTRY antimicrobial surveillance program, 1997 and 1998. Clin Infect Dis. 2000;30(3):454-60. https://doi.org/10.1086/313710 PMID: 10722427

28. Allegranzi B, Bagheri Nejad S, Combescure C, Graafmans W, Attar H, Donaldson L, et al. Burden of endemic health-careassociated infection in developing countries: systematic review and meta-analysis. Lancet. 2011;377(9761):228-41. https://doi. org/10.1016/S0140-6736(10)61458-4 PMID: 21146207

29. European Centre for Disease Prevention and Control (ECDC). Surveillance of antimicrobial resistance in Europe 2017. Annual Report of the European Antimicrobial Resistance Surveillance Network (EARS-Net). Stockholm: ECDC; 2018. Available from: https://ecdc.europa.eu/sites/portal/files/documents/EARSNet-report-2017-update-jan-2019.pdf

\section{License, supplementary material and copyright}

This is an open-access article distributed under the terms of the Creative Commons Attribution (CC BY 4.0) Licence. You may share and adapt the material, but must give appropriate credit to the source, provide a link to the licence and indicate if changes were made.

Any supplementary material referenced in the article can be found in the online version.

This article is copyright of the authors or their affiliated institutions, 2019. 